
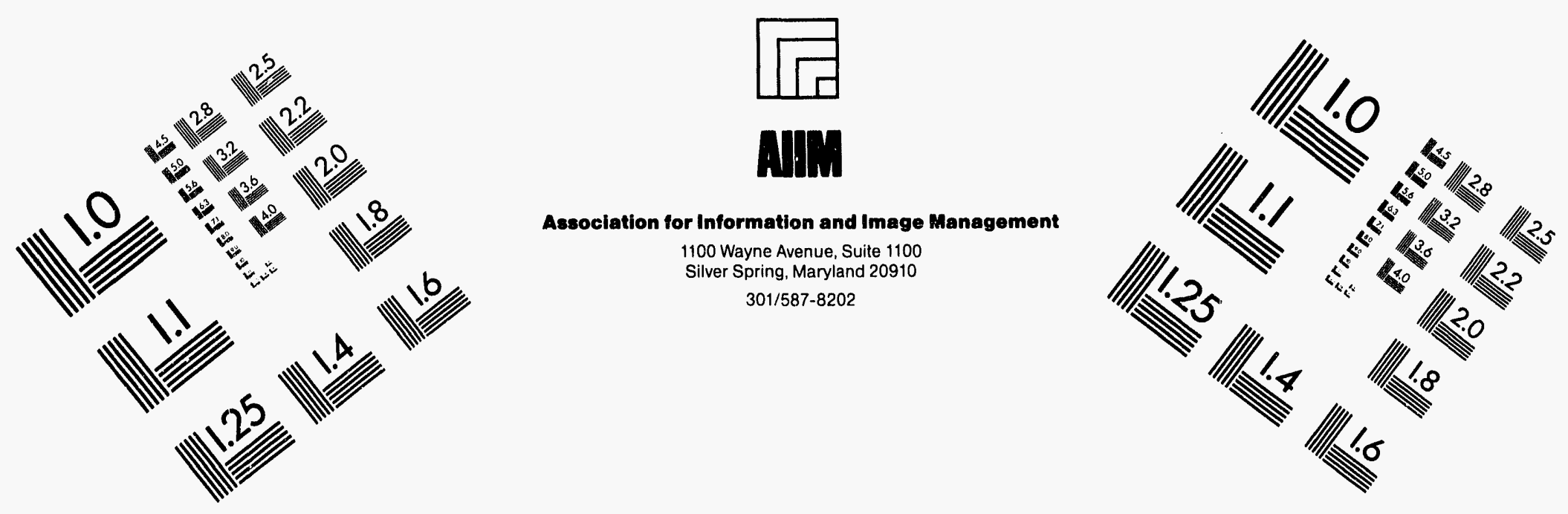

Centimeter

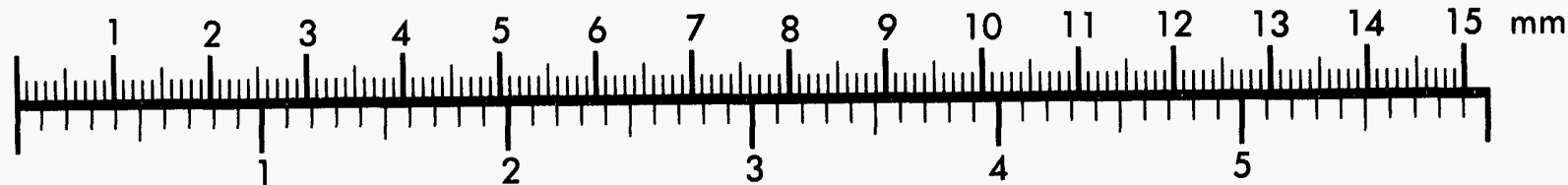
Inches
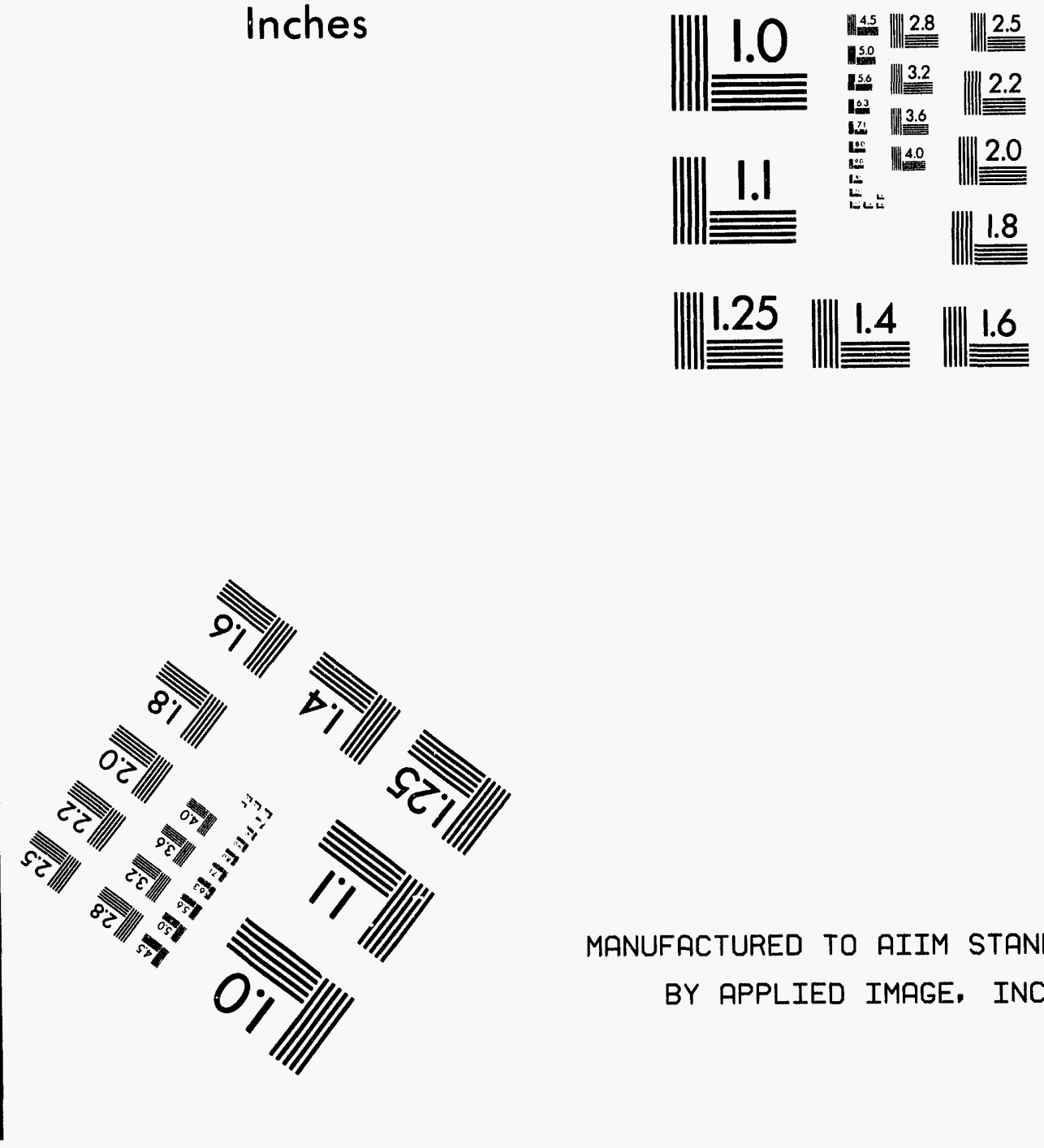

MANUFACTURED TO AIIM STANDARDS

BY APPLIED IMAGE, INC.

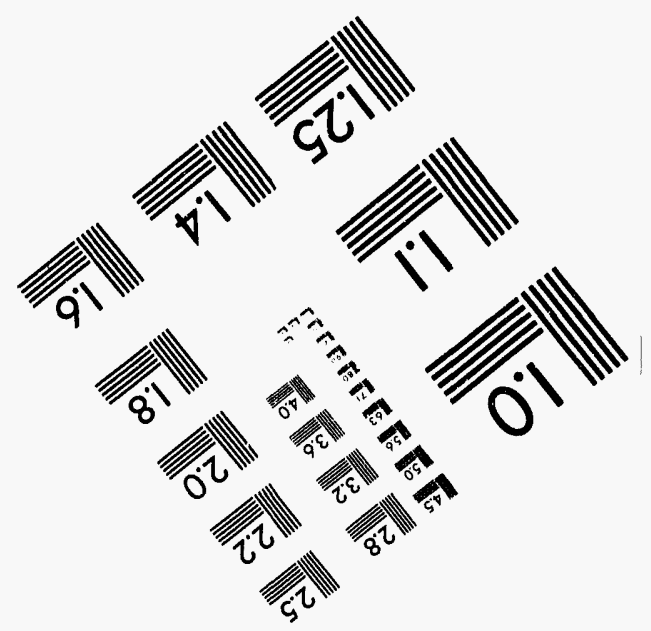



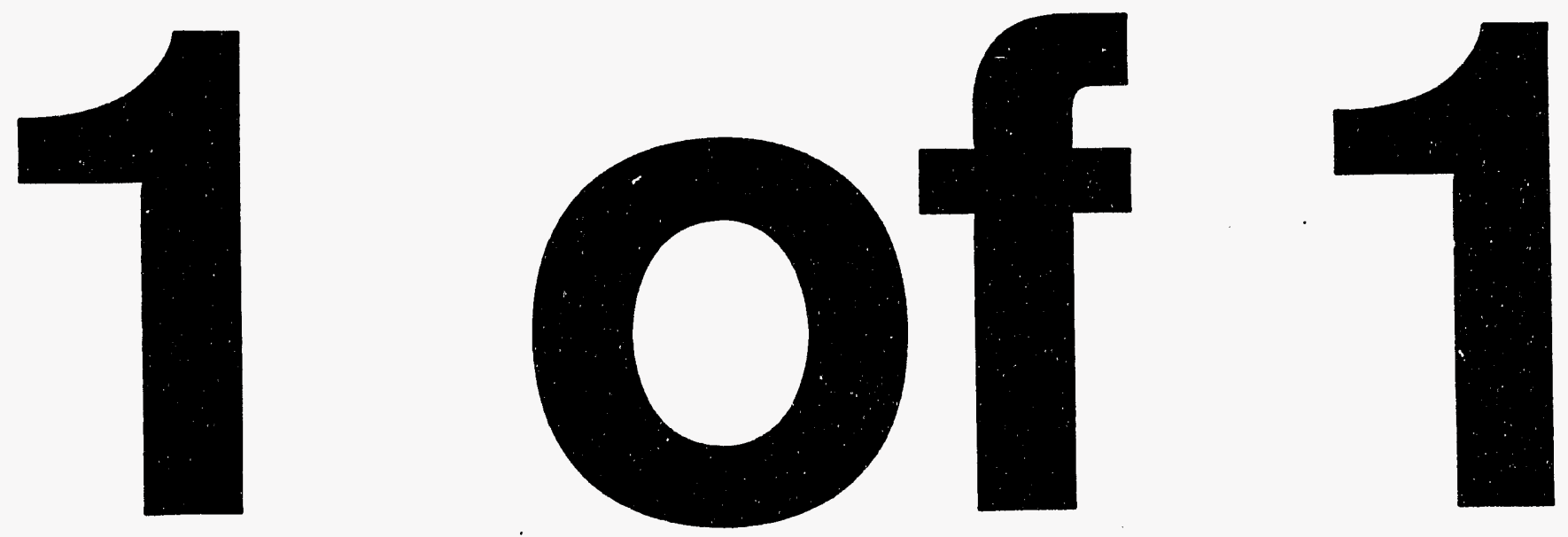
UCRL-JC-117810

PREPRINT

\title{
Fabrication and Testing of a Silicon Immersion Grating for Infrared Spectroscopy
}

\author{
Paul J. Kuzmenko \\ Dino R. Ciarlo \\ Charles G. Stevens
}

This paper was prepared for submittal to SPIE's International Symposium on Optics,

Imaging, and Instrumentation,

San Diego, California

July 24-29, 1994

July 25, 1994

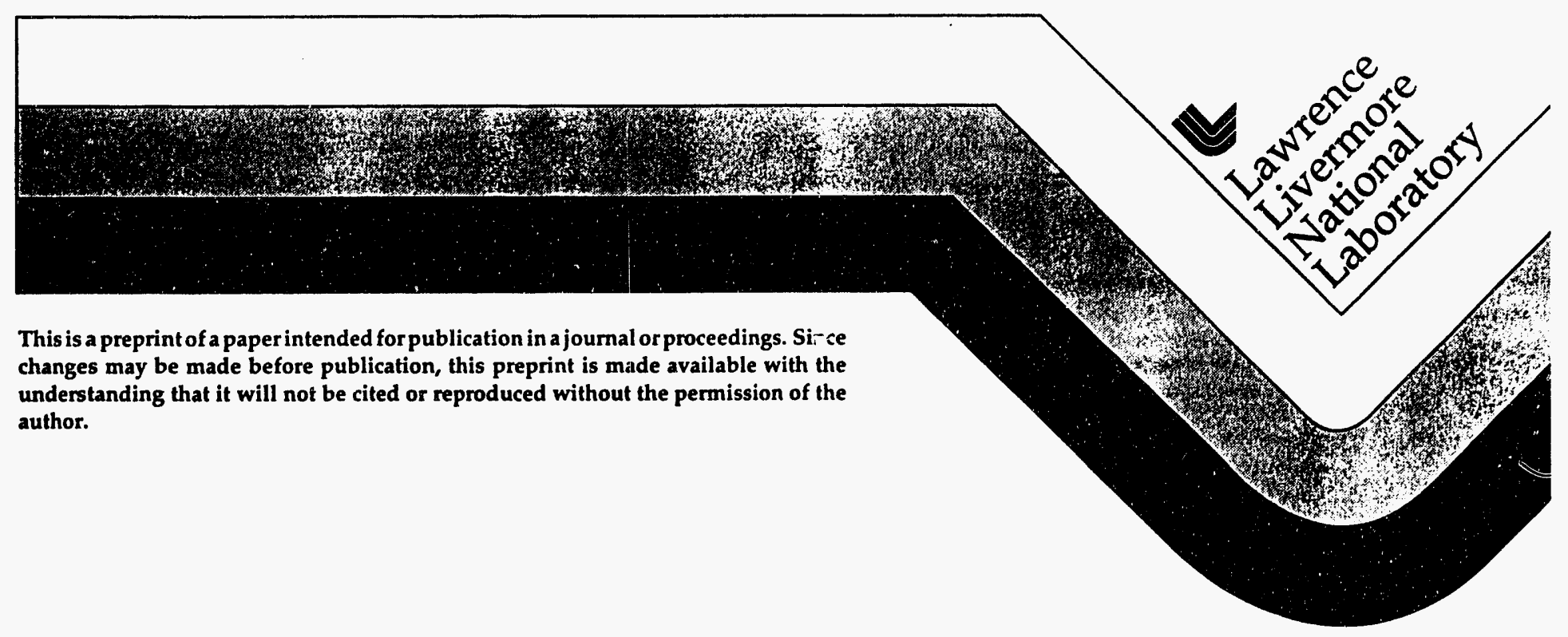




\section{DISCLAIMER}

This document was prepared as an account of work sponsored by an agency of the United States Government. Neither the United States Government nor the University of California nor any of their employees, makes any warranty, express or implied, or assumes any legal liability or responsibility for the accuracy, completeness, or usefulness of any information, apparatus, product, or process disclosed, or represents that its use would not infringe privately owned rights. Reference herein to any specific commercial product, process, or service by trade name, trademark, manufacturer, or otherwise, does not necessarily constitute or imply its endorsement, recommendation, or favoring by the United States Government or the University of California. The views and opinions of authors expressed herein do not necessarily state or reflect those of the United States Government or the University of California, and shall not be used for advertising or product endorsement purposes. 
Fabrication and testing of a silicon immer rion grating for infrared spectroscopy

Paul J. Kuzmenko, Dino R. Ciarlo and Charles G. Stevens

Lawrence Livermore National Laboratory

P.O. Box 808, Livermore, CA 94551

\begin{abstract}
Recent advances in silicon micromachining techniques (e.g. anisotropic etching) allow the fabrication of very coarse infrared echelle gratings. When used in immersion mode the dispersion is increased proportionally to the refractive index. This permits a very significant reduction in the overall size of a spectrometer while maintaining the same resolution. We have fabricated a right triangular prism $(30 \times 60 \times 67$ $\mathrm{mm}$ with a rectangular entrance face $30 \times 38 \mathrm{~mm}$ ) from silicon with a grating etched into the face of the hypotenuse. The grating; covers an area of $32 \mathrm{~mm}$ by $64 \mathrm{~mm}$ and has a $97.5 \mu \mathrm{m}$ periodicity with a blaze angle of $63.4^{2}$. The groove surfaces are very smooth with a roughness of a few nanometers. Random defects in the silicon are the dominant source of grating scatter $(\approx 12 \%$ at $3.39 \mu \mathrm{m})$. We measure a grating ghost intensity of $1.2 \%$. The diffraction peak is quite narrow, slightly larger than the Airy disc diameter at F/12. However due to wavefront aberrations, perhaps $15-20 \%$ of the diffracted power is in the peak with the rest distributed in a diameter roughly five times the Airy disc.
\end{abstract}

Keywords: immersion grating, mid-infrared, echelle grating, infrared spectrometer, etched grating, silicon

\title{
1. INTRODUCTION
}

The motivation for this work on micromachined silicon gratings comes from the development at LLNL of a mid-infrared spectrometer for remote sensing of atmospheric chemicals. (Full details on the spectrometer are presented in two other papers at this conference ${ }^{1,2}$ so only a brief description will be given here.) Designed to operate in the atmospheric windows from 2.3 to $2.5 \mu \mathrm{m}$ and 3.0 to $4.2 \mu \mathrm{m}$ this instrument uses an echelle grating operating in high order to achieve resolution at the pressure broadened limit, roughly 0.1 $\mathrm{cm}^{-1}$. The orders are evenly spread across a 2-D InSb focal plane array by a prism cross disperser. To capture the full atmospheric spectrum without gaps, one must match one order of the grating with one row of the InSb array. For a $256 \times 256$ element array, achieving $0.1 \mathrm{~cm}^{-1}$ resolution requires a grating free spectral range of roughly $25 \mathrm{~cm}^{-1}$. This implies a groove periodicity of just a few lines per millimeter. Commercially available gratings are ruled no coarser than 23 lines per millimeter so it was necessary to explore other fabrication options.

The existence of anisotropic etchants for single-crystal silicon has been known since the 1950's and the technology has been well developed both for microelectronic and micromechanical applications. Gratings have been etched on silicon wafers for nearly 20 years ${ }^{3}$ although these have generally been of small area (of order $1 \mathrm{~cm}^{2}$ ) and with less stringent performance than that demanded by high resolution spectroscopy.

Silicon has good optical, mechanical and thermal properties ${ }^{4}$. The semiconductor industry produces large quantities of very high purity, single-crystal material which ensures good optical homogeneity. For wavelengths in the 2 to 5 micron range, the optical absorption is very low. It can be polished readily and is hard enough to resist scratching. Mechanically, silicon is strong and light although somewhat brittle. Its thermal conductivity is high and the thermal expansion is low .

It has been known for some time that operating a diffraction grating immersed in a medium of refractive index $\mathrm{N}$ increases the dispersion by a factor $\mathrm{N}$ over the same grating in air $5,6,7$ (see figure 1). This is due to the angle multiplication from Snell's law at the air interface. The wavelength in the medium is divided by $\mathrm{N}$ relative to its value in air. Therefore the grating length measured in wavelengths and hence the spectral resolution are multiplied by $\mathrm{N}$. Similarly the grocve spacing measured in wavelengths increases by $\mathrm{N}$ and therefore the free spectral range is reduced by $\mathrm{N}$.

These properties of an immersion grating offer important benefits. For a fixed size grating, dispersion can be increased by a factor $\mathrm{N}$ or for a fixed value of dispersion the linear dimensions of the grating can be 
reduced by a factor $\mathrm{N}$. Since the size of the collimated beam is reduced along with the grating, the sizes of other optics in the spectrometer are reduced as well. For silicon $N \approx 3.4$, so dramatic reductions in size and weight may be achieved. These factors may be critical in the design of cryogenic, airborne or spaceborne instruments.

Although the immersed grating concept has betil known for years and the potential benefits understood, fabrication technologies have lagged behind. Only recently have the first efforts been made. Three groups in the astronomical instrumentation community have reported work in this area. Wiedemann and Jennings 8,9 etched a 50 line/mm grating on a $2 \mathrm{~cm}$ thick block of silicon cut from a $7.5 \mathrm{~cm}$ diameter ingot. The silicon was cut at an $8.7^{2}$ angle to the (100) crystal plane to give the grating a $63.4^{\circ}$ blaze angle. Two entrance faces were cut parallel to the (110) direction to allow the grating to be operated in immersion mode. When tested with a $1.15 \mu \mathrm{m}$ HeNe laser, the location of the diffraction orders was as expected. They observed ghosts around the diffraction peaks with a $2 \%$ relative amplitude. This was attributed to periodic error in the grating mask. Considerable scattered light was seen between grating orders, possibly due to misalignment of the grating mask with the crystal planes. Williams et. al. 10 etched 20 line/mm patterns onto $4 \mathrm{~cm}$ diameter $(100)$ oriented silicon wafers. They observed very smooth groove surfaces under a scanning electron microscope (SEM). Graf et. al. ${ }^{11}$ etched 40 line/mm grooves into (100) wafers and measured the diffraction efficiency with a $\mathrm{CO}_{2}$ laser. Although not tested in immersion, the grating efficiency with the laser incident from the air side matched calculations.

The purpose of this paper is to describe what we have leamed at LLNL in our first attempt at fabricating an immersion grating suitable for a compact remote sensing spectrometer. The first section discusses the fabrication procedure and the tolerances needed to achieve a high performance grating. Next are details of the grating characterization including optical and electron microscopy, interferometric tests of surface figure, and operation in a spectrometer to measure ghosts, scatter, and the focal plane intensity distribution. Foliowing that is a discussion of the test results and plans for improvements in the fabrication of future immersion gratings.

\section{FABRICATION}

The critical technologies for grating fabrication, namely optical lithography and anisotropic etching, have been well developed in the semiconductor industry. Additionally the microelectronics group at LLNL has had 15 years of experience in fabricating mechanical structures in silicon and has etched small gratings in the past. As a result we decided to bypass the step of etching practice gratings on standard silicon wafers and go straight to a silicon disc large enough to cut out a full scale immersion grating in the form of a right triangular prism. This would allow us to directly address the issues of working with a large non-standard piece of silicon.

The present grating in our prototype spectrometer is $22 \mathrm{~cm}$ long by $11 \mathrm{~cm}$ wide (the actual ruled area is $20.6 \times 10.2 \mathrm{~cm}$ ) and is ruled at 23.2 lines $/ \mathrm{mm}$ with a blaze angle of $63.4^{2}$. Taking a nominal refractive index of 3.42 for silicon the dimensions of the equivalent immersion grating shoulc be $3.2 \times 6.4 \mathrm{~cm}$. This will easily fit on a 3 inch diameter piect of silicon. The thickness required to fit a full prism is just over one inch. To attain a $63.4^{\circ}$ blaze angle the surface to be etched must lie at an $8.7^{\circ}$ angle to the (100) crystal plane tilted toward the (011) plane.

We purchased a standard photolithography mask from DuPont Photomasks, Inc. It was a $10 \mathrm{~cm}$ square, 2.4 $\mathrm{mm}$ thick glass piece coated with $100 \mathrm{~nm}$ of chromium. The pattern is written directly on the chromium with a computer controlled electron beam. We chose a pattern of $12 \mu \mathrm{m}$ wide stripes with a $97.5 \mu \mathrm{m}$ periodicity, giving a desired free spectral range of $17 \mathrm{~cm}^{-1}$. The mask also included alignment marks near the edges.

This mask was designed for use with a positive photoresist (i.e. resist exposed to UV through the mask gets removed) because these have superior resolution and are more resistant to pinholes than negative photoresists.

We had some difficulty in procuring the silicon discs. There are two types of potential vendors. Growers of silicon for infrared optics work with standard crystal orientations and generally do not have the equipment to determine off-axis cuts. They can grind the crystal very flat but leave subsurface damage that interferes with the anisotropic etching. Growers of semiconductor grade silicon can supply arbitrary orientations and 
perform a special polish to remove damaged layers but are not equipped to get sufficient flatness in nonstandard thicknesses. We ordered our silicon ( 3 inch diameter, 1.1 inch thick, p-type, resistivity $>100$ $\Omega \mathrm{cm}$ ) from a semiconductor supplier, but the surfaces had 15 to 20 waves of sphericity and had to be reground in-house. The pieces were also elliptical, having apparently been sliced from a (100) boule.

The high refractive index of the silicon causes the fabrication tolerances to be tight. Stroke 12,13 provides a good discussion of the effects of errors on grating performance. Deviations from flatness in the grating substrate cause aberrations in the diffracted beam. For a grating user in Littrow mode the wave-front aberration is twice the cosine of the blaze angle multiplied by the substrate flatness error. Stroke recommends an upper limit of one wave of aberration for general spectroscopy, but less than $\lambda / 5$ for high resolution work. For example, if our silicon substrate were flat to $\lambda / 4$ at $632.8 \mathrm{~nm}$ the wave-front aberration from the air side would be about $140 \mathrm{~nm}$ or roughly $\lambda / 20$ at $3 \mu \mathrm{m}$. However when used in immersion, the effective wavelength in the silicon is diminished by the refractive index of 3.4, giving a wave-front error of $\lambda / 6$ at $3 \mu \mathrm{m}$.

The LLNL optics shop polished the silicon to $\lambda / 10$ in the visible using conventional techniques. However, this damages the crystalline structure of the silicon beneath the surface to a depth of a few times the size of the smallest abrasive used. To eliminate this damage, a final polish with a high $\mathrm{pH}$ slurry of colloidal silica is used to remove the top 1 to $2 \mu \mathrm{m}$. The flatness was then rechecked and found to better than $\lambda / 8$ peak to valley. Repeating the calculation in the previous paragraph, the immersion grating wave-front aberration at the shortest wavelength of use $(2.3 \mu \mathrm{m})$ is determined to be better than $\lambda / 9$.

Once the proper silicon has been obtained the steps in fabricating the grating are as follows. First a $90 \mathrm{~nm}$ layer of silicon nitride is deposited on the silicon to serve as a chemically resistant mask for the etch process. Next, a positive photoresist is applied with a spinner. The grating pattern is contact printed on the resist, and the resist is developed leaving windows in the resist where exposed to UV light. The exposed nitride is then plasma etched down to the silicon. The disc is placed in an anisotropic etch (44\% $\mathrm{KOH}$ in water at $40^{\circ} \mathrm{C}$ ) which etches downward at about $5 \mu \mathrm{m}$ per hour. The etch essentially stops when it reaches the (111) planes which form the grooves. It is very important to achieving high anisotropy in the etch and smooth groove faces that the mask be precisely aligned to the (111) planes with an accuracy of better than $0.1^{\mathrm{Q}}$. Flat surfaces are typically ground onto silicon by vendors to indicate crystal orientation, but the accuracy is typically $\pm 1^{2}$. The procedure ${ }^{14}$ we used was to etch a splay pattern consisting of radial lines spaced $0.1^{2}$ apart in an unused part of the silicon surface. The groove with the cleanest surface indicated the direction most closely aligned with the crystal axis. This was used to align the pattern for the grating (see figure 2). After etching, gold is deposited on the groove faces to enhance reflectivity ( $200 \mathrm{~nm}$ of Au over 10 $\mathrm{nm}$ of $\mathrm{Cr}$ for adhesion). Finally, the prism is cut out of the disc using a grinder and the entrance face is polished flat. To avoid spurious reflections the entrance face should be cut at a slight angle to the groove faces and should be antireflection coated.

Some problems arose during the first attempt at grating fabrication. Hardware designed for processing wafers generally did not work well with the large silicon disc. For example, the disc was too thick to fit into the mask aligner. So the photomask was mounted in an aluminum frame and manually aligned under a microscope before exposing the resist.

Standard photoresist is optimized for wafers spinning at 4000 to 5000 RPM. The mass and ellipticity of the silicon caused the spinner to vibrate severely at only 1500 RPM resulting in an overly thick resist layer. Grinding the silicon into a right circular cylinder reduced vibrations, but did not greatly increase the spin rate. We have since found a low viscosity resist (AZ1350B) that yields satisfactory thicknesses at low RPM's. The photoresist initially did not adhere well to the silicon nitride. Baking the silicon at high temperature to slightly oxidize the nitride gave satisfactory results. Recently we have used a standard wafer primer (HMDS) on some test pieces and adhesion is excellent.

One of the consequences of the overly thick resist layer was that the photosensitive material was underexposed relative to a more nominal thickness. After development, the resist was not removed cleanly down to the nitride layer, so the development was resumed until some of the resist began to lift off in areas where it was supposed to adhere. At this point development was halted, the disk was baked to harden the remaining resist and plasma etching of the nitride was begun. Not all the exposed resist was removed during the development process so the plasma etch was allowed to continue until it had gone through this 
excess resist and the underlying nitride. As a result some areas of the disc were over etched into the silicon (see figure 3). Despite these difficulties the finished grating performed reasonably well. Details of the testing will be found in the next section.

\section{TESTING}

Tests of a grating can be broken up into two categories one of which is essentially optical inspection and the other is performance. The first optical inspection tests were performed during fabrication. Photographs were taken under a microscope before the $\mathrm{KOH}$ etch and at intervals during the etch. Figures 4 and 5 show examples of these. To the limit of the optical magnification used, the mask edges are very straight and the (111) planes forming the groove faces are perfectly smooth. After the etch was completed but before coating, scanning electron microscope (SEM) photographs at varying magnifications were taken of the grooves (see figures 6 and 7). Just as in the optical photographs the grooves were found to be perfectly smooth and flat. At the very highest magnification $(50,000 \mathrm{x})$ a few random blobs of perhaps $100 \mathrm{~nm}$ diameter and $10 \mathrm{~nm}$ thick could be seen on the groove faces. Under visual inspection, however, point defects could be seen scattered across the etched surface (see Figure 8).

Grating function can be qualitatively demonstrated by directing a visible HeNe beam onto the grooves. If set up in Littrow mode, a white card near the laser output may be used to display the diffracted beams. Since the grating is so coarse many orders are visible. One also notices a fair amount of scattered light filling a $1.7^{\circ}$ diameter circle centered on the specular direction from the groove face. Scalar scattering theory 15 predicts a total integrated scatter of $1-\exp -(4 \pi \sigma / \lambda)^{2}$, where $\sigma$ is the RMS surface roughness and $\lambda$ is the wavelength of incident light. Based on the roughness observed by the SEM of a few nanometers, one would calculate a total scatter of perhaps $1 \%$. The fact that we see considerably more scatter (roughly 30 to $35 \%$ of incident power) and that its angular distribution is sharply peaked leads one to conclude that the observed scatter is not due to nanometer-scale groove roughness, but rather to the presence of surface defects. While still in the same setup, it is instructive to look at the diffraction pattern from the flat tops of the grooves. These will be found at twice the blaze angle $\left(\approx 127^{\circ}\right)$ from the incident beam. Since these surfaces were coated with silicon nitride and never etched, they maintain the smoothness of the initial polish. Very clean diffracted spots are observed with no visible scatter.

A procedure recommended by Stroke 12 for measuring wave-front distortion is to place the grating in one arm of a Twyman Green interferometer, tilted at the angle of operation so that the diffracted rays interfere with the reference beam. This produces an interferogram of the diffracted beam that displays aberrations in the wave front. The procedure was carried out on our immersion grating using a Zygo model XPE interferometer system operating at $632.8 \mathrm{~nm}$. Since the silicon does not transmit at this wavelength, the grating was turned around so that the grooves were illuminated from the air side and not in immersion. This would fairly represent the diffracted wave front in immersion except for the effect of possible inhomogeneities in the silicon and deviations from flatness in the entrance face. The grating interferogram is presented in figure 9 and that of the prism entrance face in figure 10. Correcting for the refractive index of silicon and the longer wavelengths that the grating will be used at in the spectrometer, the grating aberrations in immersion mode will range from 50 to $90 \%$ of those shown here. The grating interferogram does not look very good. There appears to be about one wave of aberration over the center of the grating, but over about 20 to $25 \%$ of the surface the wavefront is too aberrated for the Zygo to track. These regions appear as dropouts when it tries to generate a profile plot. This was likely due to the penetration into the silicon of the plasma etch over parts of the surface, effectively making the surface less flat than it had been after polishing.

Another test performed with the Zygo was to take an interferogram (see figure 11) of the beam diffracted from the flat tops of the grooves. The groove tops are part of the surface of the original silicon disc, masked by silicon nitride and untouched by the etch. If the silicon was not distorted during processing, the groove tops should maintain the flatness of the original disc (better than $\lambda / 8$ ). However the interferogram shows the surface to be cylindrically concave by about $\lambda / 4$, with the narrow ends of the rectangular grating pattern curved upward. The end with the thinnest silicon had more curvature. This curvature was likely due to differential contraction of the gold coating relative to the silicon substrate.

The easiest way to test grating performance is to insert it into a spectrometer. Our existing prototype spectrometer has an $F / 10,100 \mathrm{~cm}$ focal length collimator that produces a $10 \mathrm{~cm}$ beam. After dispersion the 
beam is focused into the InSb array with F/3.5 optics. We adapted the cover for the ruled grating to mount the immersion grating. Due to the much smaller size of the silicon grating, the collimator effectively operates at F/33 and final optics at about $F / 12$. This results in a loss of throughput and a larger diffraction limited spot on the focal plane array. To test the new grating, light from a $3.39 \mu \mathrm{m}$ HeNe laser was injected into the spectrometer using a zirconium fluoride glass multimode optical fiber. The $100 \mu \mathrm{m}$ diameter fiber core serves as the entrance aperture. Geometrical optics predicts an image size of $35 \mu \mathrm{m}$ diameter at the focal plane. However the Airy disc for an F/12 system at $3.39 \mu \mathrm{m}$ is nearly $100 \mu \mathrm{m}$, so this will be the dominant component of image size in the absence of aberrations. The present focal plane array has $50 \mu \mathrm{m}$ square pixels, so a diffraction limited spot should cover a $2 \times 2$ block of pixels. The InSb focal plane array is part of a commercial infrared imaging system (ImagIR from Santa Barbara Focalplane). The output of each pixel in the 128x128 array is digitized by a 12 bit A/D. Provision is made for flat-fielding and nonuniformity correction. Compiete video frames can be captured and stored digitally for later analysis.

The first test with the immersion grating was to obtain the minimum focal spot in the image plane and examine the intensity distribution. This provides a direct indication of the wavefront aberration due to the immersion grating. Figures 12 and 13 show the intensity distribution and give the profile through the peak. The Airy disc due to diffraction should be a $2 \times 2$ block of pixels. We see a narrow peak slightly wider than this, but there is substantial intensity in a broad pedestal around the peak. By taking the ratio of the summed intensity of brightest 4 pixels to the summed intensity above thermal background in the roughly $10 \times 10$ pixel area comprising the pedestal, we estimate the fraction of the diffracted power in the main peak to be about $18 \%$. This is consistent with the aberrations seen in the interferograms of the groove surfaces and the entrance face.

Another important test is to look for grating ghosts caused by periodic errors in groove position. We saw a pair of ghosts located symmetrically around the main spot at a distance of $5.1 \mathrm{~mm}$ (102 pixels). They had peak intensities about $1.2 \%$ that the main spot. Using the standard equations for Rowland ghosts 12,13 the amplitude of the periodic error is calculated to be $0.066 \mu \mathrm{m}$ and the periodicity is $520 \mu \mathrm{m}$. This agrees well with some information given by the mask vendor. They used 1024 address units to write the grating. For this job each unit was $0.5 \mu \mathrm{m}$, giving a total of $512 \mu \mathrm{m}$ before the pattern must be stepped. Hence the periodic error would seem to come from the pattern stepping during the mask generation.

One observes scattered light on the captured images, but it is difficult to accurately quantify. The main reason is that the focal plane array has about a $1^{2}$ full angle field of view and any light scattered at large angles will not be detected by the array. However, our experience shining a visible $\mathrm{HeNe}$ on the etched surface showed that by far the majority of the scattering was within a degree or so of the specular reflection. One approach to estimating scatter is to first separate the laser scatter from the thermal background. This is done by subtracting, pixel by pixel, a frame with the laser off from a frame with the laser on. Next the contributions of the image and ghost must be removed leaving only the scatter. All the pixel intensities in the difference frame are summed. The pixel intensities in the image and in the ghost are subtracted and the remainder represents the scatter. We have included the contribution from all pixels within 5 pixels of the image and ghost peaks into the image and ghost sums. Light that is detected within 5 pixels of a peak is not true scatter, but spread in the focal spot due to wavefront aberrations. For a frame with the laser image near one comer about $3 \%$ of the photons striking that frame are scattered light. So perhaps something like $12 \%$ would be an estimate of the total scatter. One can compare this value with the externally measured scatter as follows. The effective wavelength of the $3.39 \mu \mathrm{m}$ laser inside the silicon is $3.39 / 3.42$ or roughly $0.99 \mu \mathrm{m}$. Scattering from large defects is predicted to scale as $\lambda^{-2}$ (see Stroke ${ }^{12,13}$ ). Therefore the ratio of scattered power measured externally at $633 \mathrm{~nm}$ to that measured internally at an effective $990 \mathrm{~nm}$ is $(990 / 633)^{2} \approx 2.45$. So the very rough estimate of $12 \%$ scatter measured at $3.39 \mu \mathrm{m}$ in immersion would be consistent with an external scattering of about $30 \%$ with a visible HeNe.

\section{DISCUSSION}

While our first attempt at fabricating a silicon immersion grating was not suitable for high resolution spectroscopy, several important things were learned during the fabrication and characterization processes. The major difficulties that need to be addressed are reducing the density of surface defects in the silicon and reducing the wavefront aberration. 
The presence of grating ghosts is less of a problem. Grating masks generated on interferometrically controlled ruling engines are available at about an order of magnitude increase in price over commercial semiconductor masks. These have extremely small periodic errors and would give ghost intensities in the 0.0001 to $0.001 \%$ range. It may also be possible to locate a semiconductor mask vendor that can produce the complete grating pattern without having to step and repeat.

The issue of silicon defects may be addressed in two ways: using material with lower defect density and reducing the growth of defects during processing. Anisotropic etching is a good way to locate crystal defects and there is a standardized procedure for this ${ }^{16}$. It should be possible to perform this test on asreceived silicon and repolish the best pieces to be etched into gratings. It is well known 17 that temperature cycling causes defect growth and that low temperature processing is preferred. We attempted a second grating using a thermally grown oxide as a mask (oxidization temperature $\approx 1100^{\circ} \mathrm{C}$ vs. $\approx 800^{\circ} \mathrm{C}$ for deposition of silicon nitride) but the defect density in the grooves was very high. Probably the rate of temperature change is important insofar as it contributes to internal strain. On a thin silicon wafer with presumably rapid thermal equilibration time, oxidization does not seem to cause such a problem.

There are many options for the grating fabrication process. $\mathrm{KOH}$ appears to be the best anisotropic etchant, but there are several choices for the masking layer. Although some of the earlier work $9,10,11$ was done with an oxide mask, silicon nitride is a superior material. It is harder, less prone to pinholes or scratches and is etched ${ }^{18}$ by $\mathrm{KOH}$ at $<1 \mathrm{~nm}$ per hour vs. perhaps $10 \mathrm{~nm}$ per hour at $40^{\circ} \mathrm{C}$ for oxide. The lower deposition temperature for nitride is preferable for the reduction of defects. Wet chemical etching of silicon nitride is difficult (the $\mathrm{H}_{3} \mathrm{PO}_{4}$ etchant attacks photoresist) and it is usually plasma etched. Another possibility is silicon carbide, which is harder and tougher than the nitride and also unaffected by $\mathrm{KOH}$. A recent paper 19 reports deposition at 200 to $300^{\circ} \mathrm{C}$. Silicon carbide must be plasma etched.

The main reason for the wavefront distortion seems to be a poor surface figure on the grating. This poor surface figure was apparently caused by plasma overetching. Several steps can be taken to remeriy this. We have optimized our lithography for the low resist-spinner speeds that can be achieved with large silicon discs. Now the plasma etch can start directly on the nitride without any residual photoresist. The original etching was done in a barrel plasma etcher which is not noted for uniformity of etch. We are presently experimenting with a planar plasma etcher that etches very uniformly and in a straight downward direction rather than isotropically. There are changes that can be made to the chemical composition of the etch plasma to provide up to a $15: 1$ etch selectivity for the nitride vs. the silicon ${ }^{20}$. The mix used in the barrel etcher actually had an etch preference for silicon so that the rate increased once the nitride was penetrated. These steps should help maintain on the etched grooves, the surface quality of the original polished silicon.

\section{CONCLUSIONS}

We have fabricated a silicon immersion grating in the form of a right triangular prism and tested it both with optical inspection techniques and in a spectrometer. Diffraction orders can be clearly seen with a visible HeNe laser. We estimate a scatter loss of about $12 \%$ at $3.39 \mu \mathrm{m}$ due to defects in the silicon. This may be reduced by careful material selection and by processing the silicon at lower temperatures. The diffraction peak is quite narrow, slightly larger than the Airy disc diameter at F/12. However due to wavefront aberrations, perhaps $15-20 \%$ of the diffracted power is in the peak with the rest distributed in a diameter roughly five times the Airy disc. Modifications in the etching process should preserve the surface quality and drastically cut the aberrations.

\section{ACKNOWLEDGEMENTS}

Thanks to Ben Fuchs, Mike Nichols and Bob Raether of the L.LNL Optics shop for their skilled grinding and polishing of the silicon and to Marsha Kellam for produci ig the interferograms. A special debt is owed to Milt Bell for mounting and polishing the extremely fragil: infrared optical fiber. Thanks to Izzy Lewis for clarifying the relation between wavefront aberration and image intensity distribution. This work was performed under the auspices of the U. S. Department of Energy by the Lawrence Livermore National Laboratory under Contract W-7405-Eng-48. 


\section{REFERENCES}

1. C.G. Stevens, P.J. Kuzmenko, T.W. Alger and N.L. Thomas, "Echelle grating spectrograph for midinfrared remote molecular detection," this conference.

2. N.L. Thomas, S.A. Johnson and C.G. Stevens, "Design of an immersion echelle-prism mid-IR spectrograph for chemical remote sensing," this conference.

3. W.T. Tsang and S. Wang, "Preferentially etched diffraction gratings in silicon," J. Appl. Phys., vol.46, 2163-2166 (1975).

4. K.H. Hinkle, R. Drake and T. Ellis, "Cryogenic single-crystal silicon optics," Instrumentation in Astronomy VII, SPIE vol. 2198, 516-524 (1994).

5. E. Hulthén and H. Neuhaus, "Diffraction gratings in immersion," Arkiv fur Fysik, vol. 8, 343-353 (1954).

6. L. Sica, Jr., "High resolution diffraction grating," U.S. patent 4,475,792 (1984).

7. H. Dekker, "An immersion grating for an astronomical spectrograph," in Instrumentation for GroundBased Optical Astronomy, L.B. Robinson, ed., 183-188, Springer-Verlag, New York (1987).

8. G. Wiedemann and D.E. Jennings, "Immersion grating for infrared astronomy," Appl. Opt., vol. 32, 1176-1178 (1993).

9. G. Wiedemann, H. Davé and D.E. Jennings, " Immersion grating and etched gratings for infrared astronomy," Infrared Detectors and Instrumentation, SPIE vol. 1946, 622-628 (1993).

10. D.M. Williams, C.L. Thompson, G.H. Rieke and E.F. Montgomery, "Compact high-resolution IR spectrometer for the Columbus telescope," Infrared Detectors and Instrumentation, SPIE vol. 1946, 482-489 (1993).

11. U.U. Graf et. al., "Fabrication and evaluation of an etched infrared diffraction grating," Appl. Opt., vol. 33, 96-102 (1994).

12. G.W. Stroke, "Diffraction gratings," in Handbuch der Physik, vol. 29, 426-754, Springer-Verlag, Berlin (1967).

13. G.W. Stroke,"Ruling, testing and use of optical gratings for high-resolution spectroscopy," in Progress in Optics, vol.2, E. Wolf, ed., 3-72, Academic Press, New York (1963).

14. D.R. Ciarlo and D.E. Miller, "Silicon diffraction gratings for multilayer structures," Multilayer Structures and Laboratory X-Ray Laser Research, SPIE vol. 688, 163-170 (1986).

15. J.M. Bennett and L. Mattsson, Introduction toSurface Roughness and Scattering, 51-52,Optical Socicty of America, Washington, D.C. (1989).

16. ASTM F 47-88, Standard test method for crystallographic perfection of silicon by preferential etch techniques.

17. P. Van Zant, Microchip Fabrication: A Practical Guide to Semiconductor Processing, 2nd ed., 52-54, McGraw-Hill, New York (1990).

18. H. Seidel, L. Csepregi, A. Heuberger and H. Baumgärtel, "Anisotropic etching of crystalline silicon in alkaline solutions," J. Electrochem. Soc., vol. 137, 3612-3626 (1990).

19. A. Klumpp et. al., "Amorphous silicon carbide and its application in silicon micromachining," Sensors and Actuators A, vol. 41-42, 310-316 (1994).

20. D.L. Flamm and J.A. Mrcha, "Plasma etching," in The Chemistry of the Semiconductor Industry, S.J Moss and A. Ledwith, ed., 374-378, Blackie \& Son, Glasgow (1987). 
High index IR transparent materials such as Germanium $(n=4)$ and silicon $(n=3.4)$ can be used in an immersion mode to enhance grating performance by $n$.

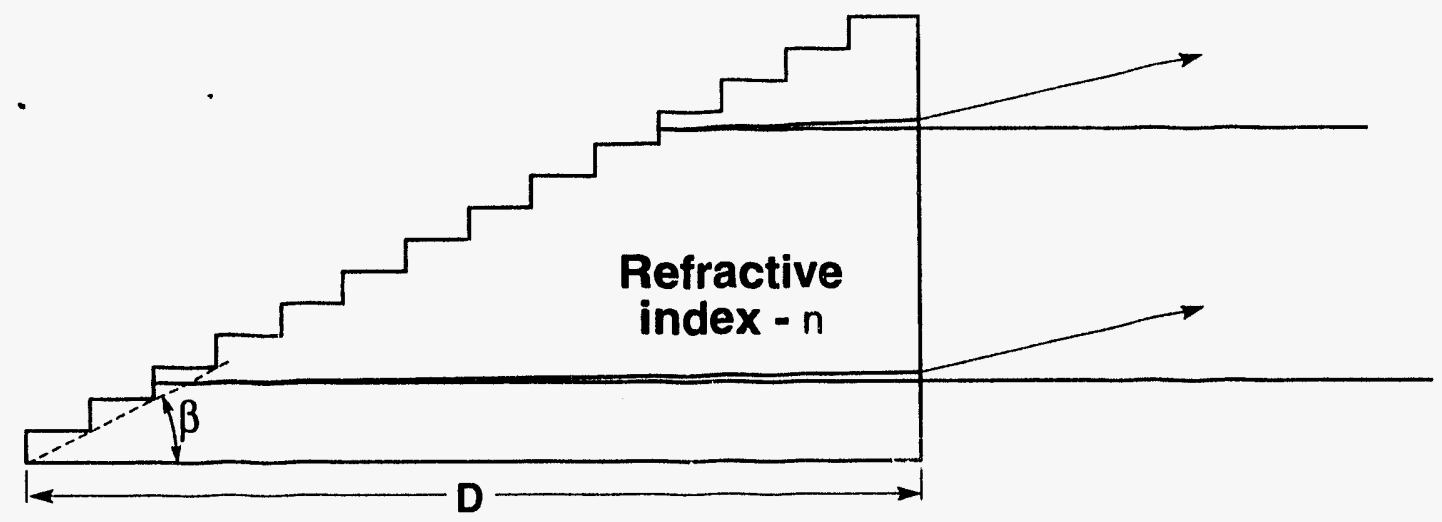

$$
\begin{aligned}
\text { Resolving power (R) } \frac{\lambda}{\Delta \lambda} & =\frac{\mathrm{nD}}{1 / 2 \lambda} \\
\text { Linear dispersion } & =\frac{n f}{(1 / 2 \lambda) \tan \beta}
\end{aligned}
$$

\section{Size of spectrometer can be reduced by a factor of $n$} while retaining resolution and light-gathering power.

Figure $1 \mathrm{IR}$ immersion gratings promise substantial improvements in dispersive instrument performance

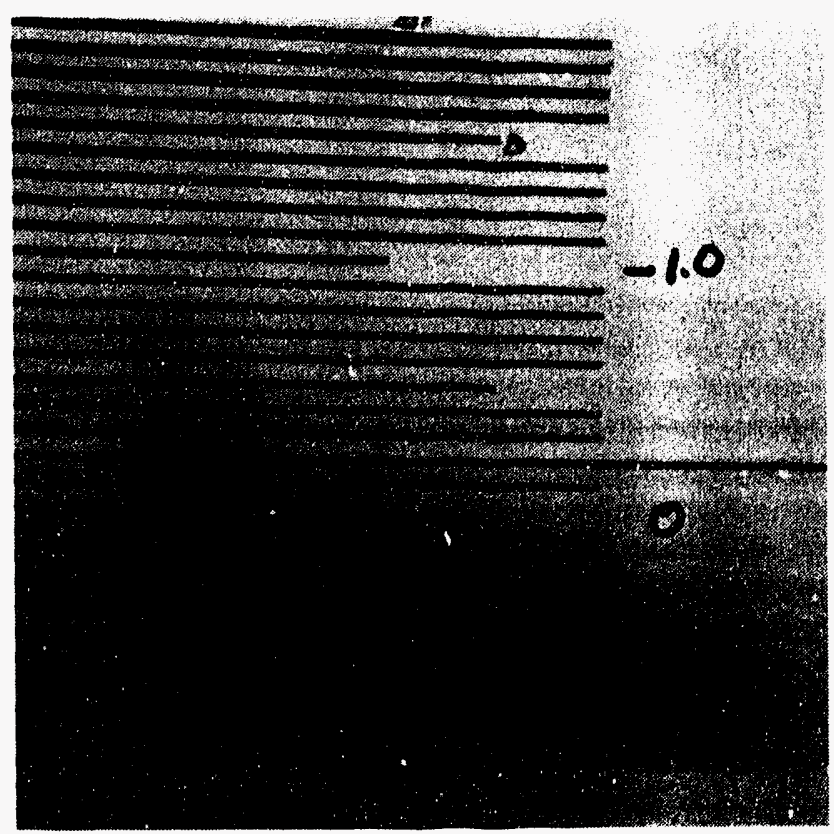

Eigure 2 Etched SPLAY pattern is used to align grating mask

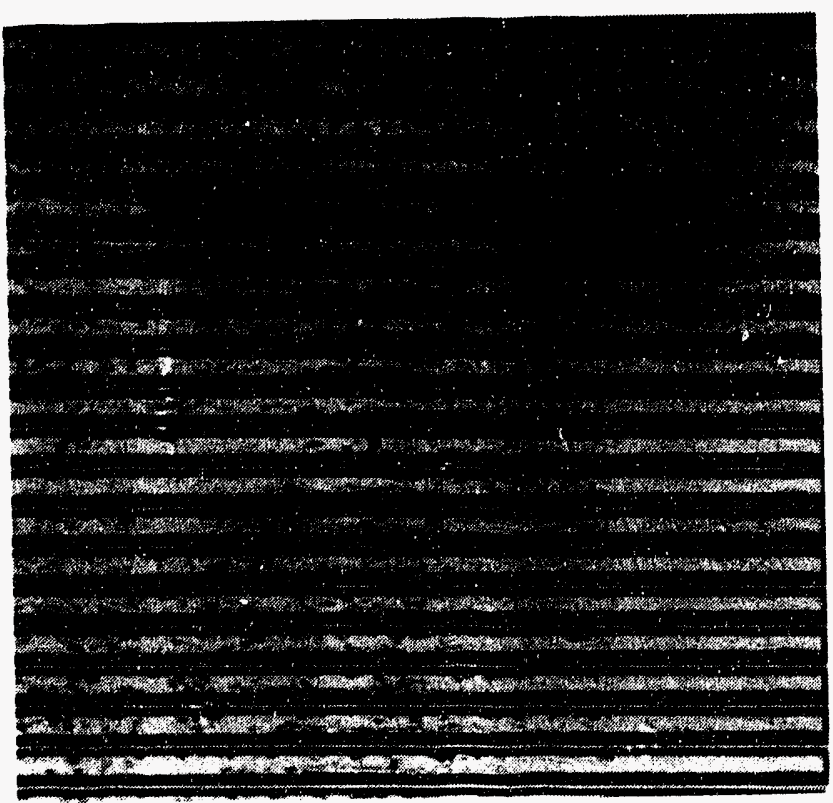

Figure 3 Damage due to plasma overetch is indicated by roughness at bottom of groove 


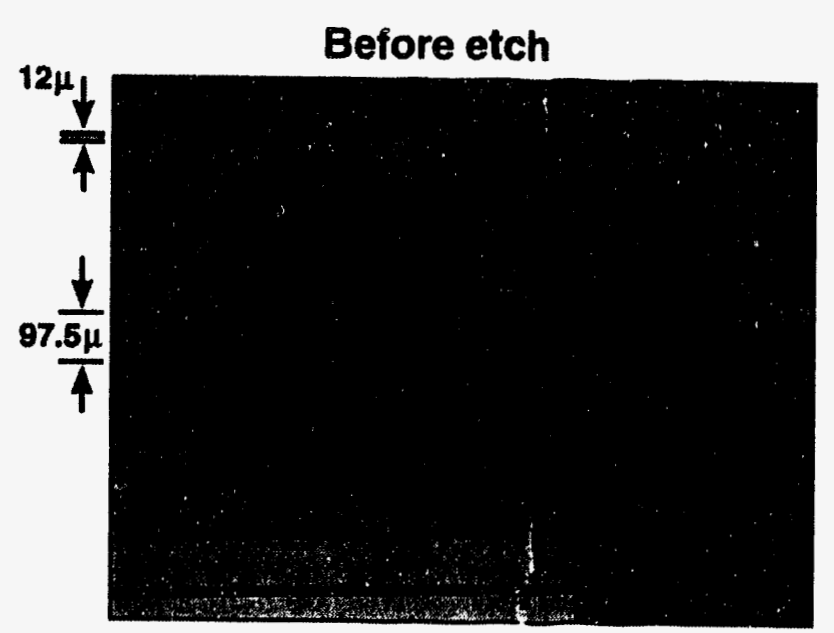

Figure 4 Masked silicon surface before anisotropic etch
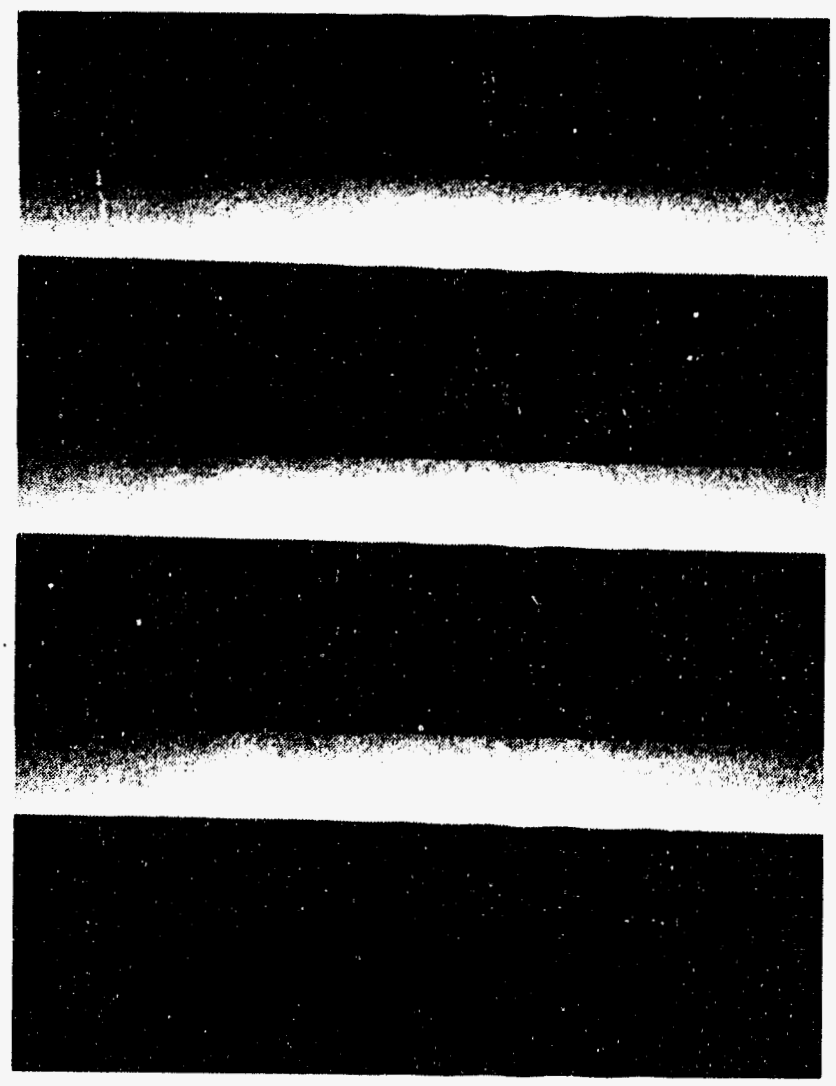

Figure 6 Groove structure at $300 \mathrm{X}$ magnification

\section{During etch}

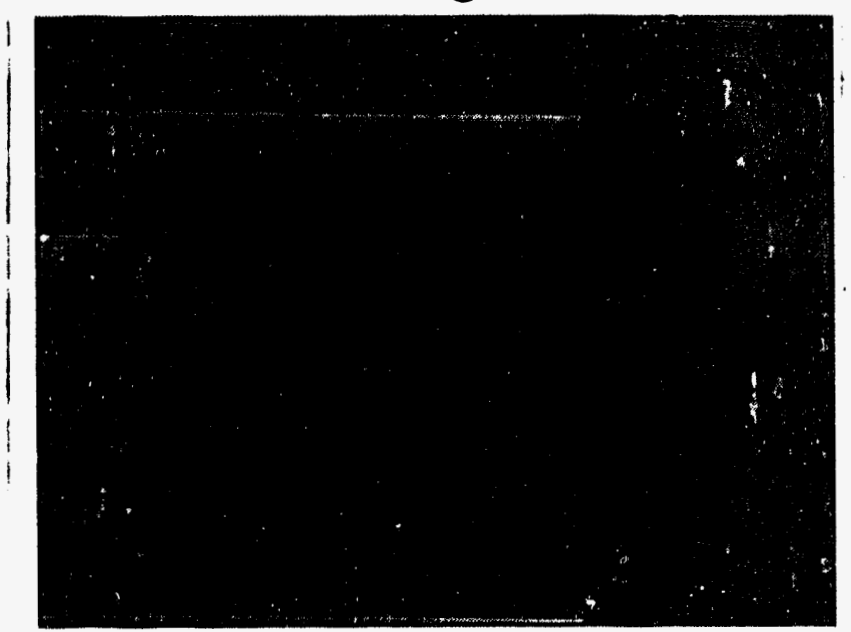

Figure 5 Silicon surface during anisotropic etch

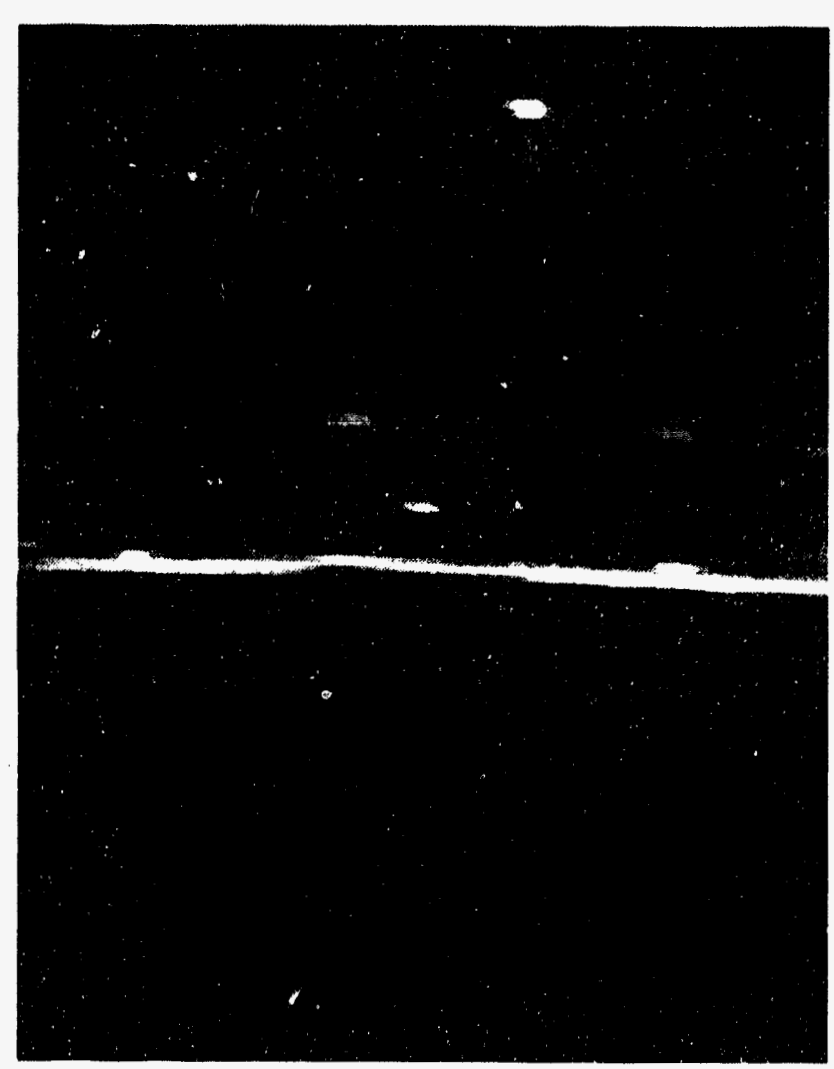

Figure7 Groove surface at 50,000X magnification. Flatness is excellent except for random "blobs" 


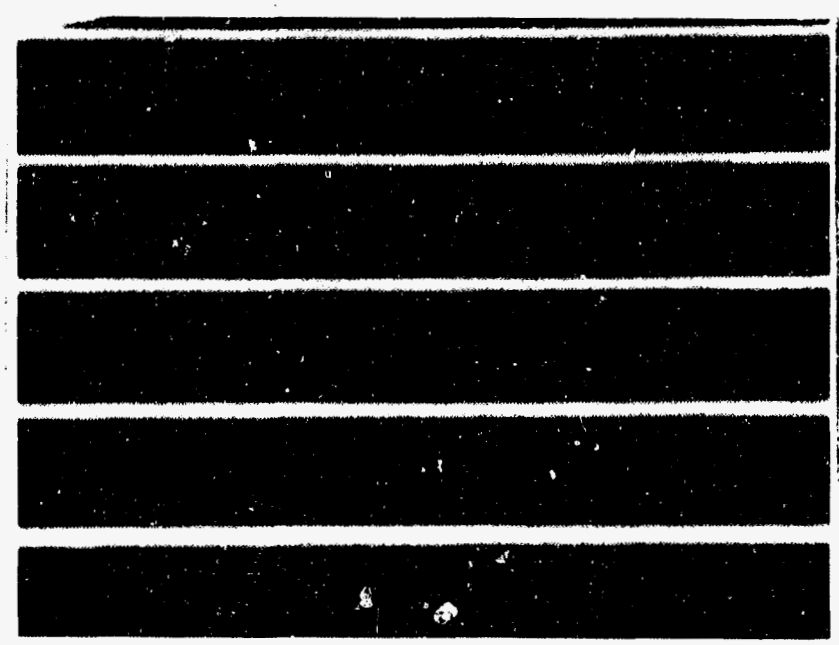

Figure 8 Silicon defect in etched groove

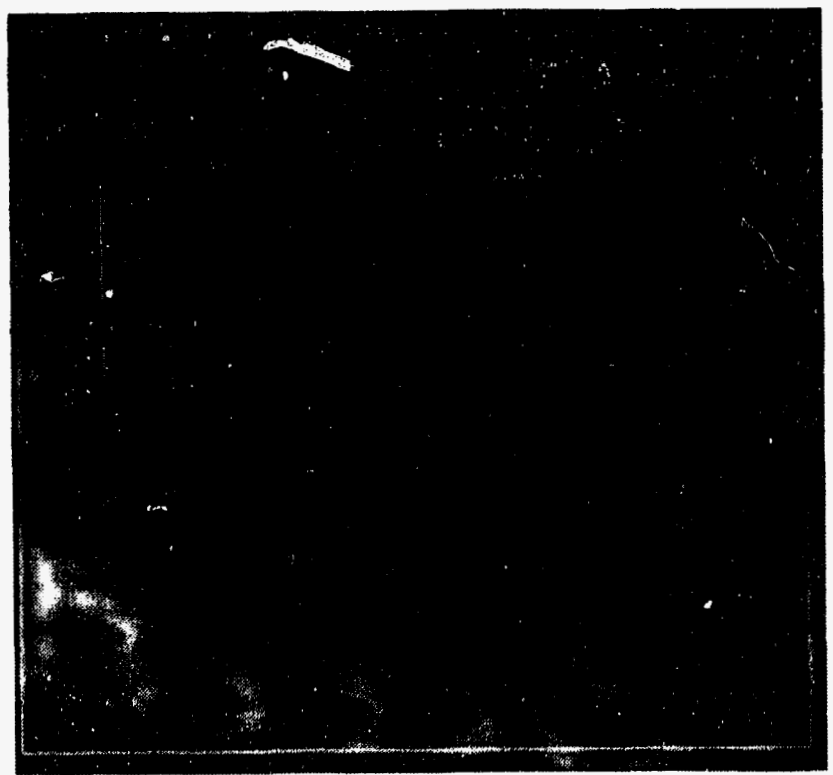

Figure 9 Interferogram of groove surfaces

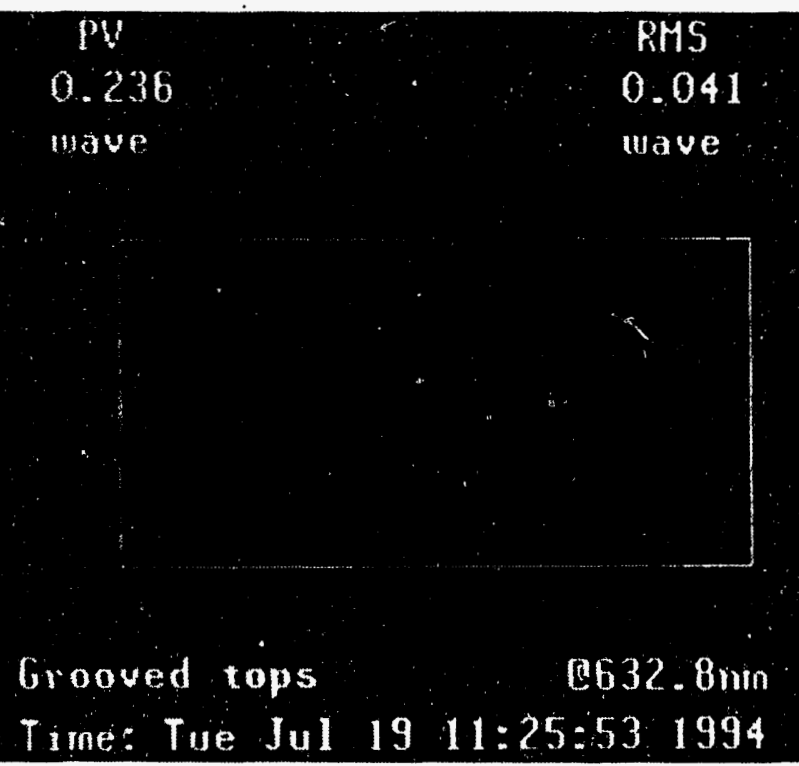

Eigure 11 Interferogram of unetched groove tops 


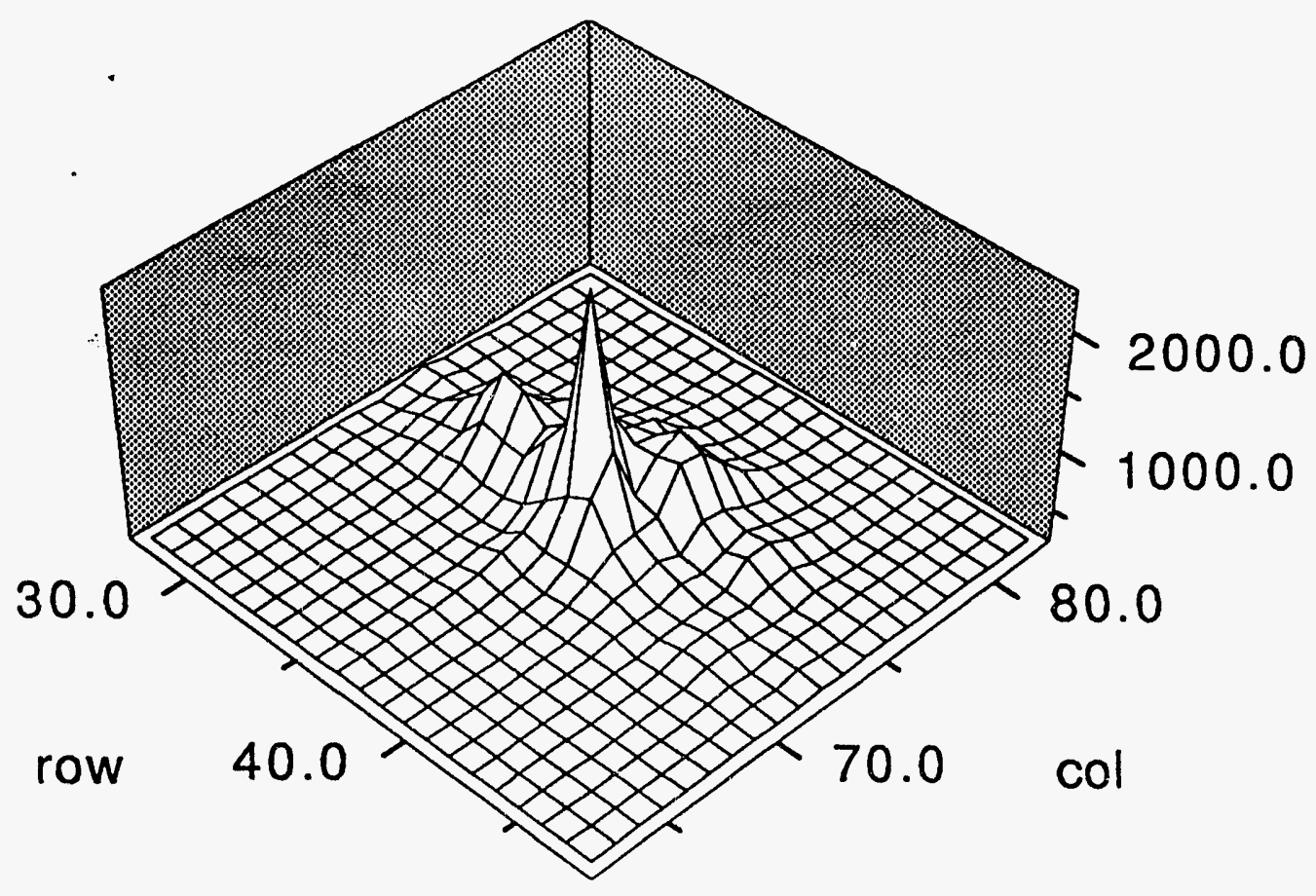

Figure $123-D$ plot of intensity in a $15 \times 15$ section of the focal plane around the peak. Spectrometer input is a $3.39 \mu \mathrm{m}$ laser injected by a $100 \mu \mathrm{m}$ core fiber. 


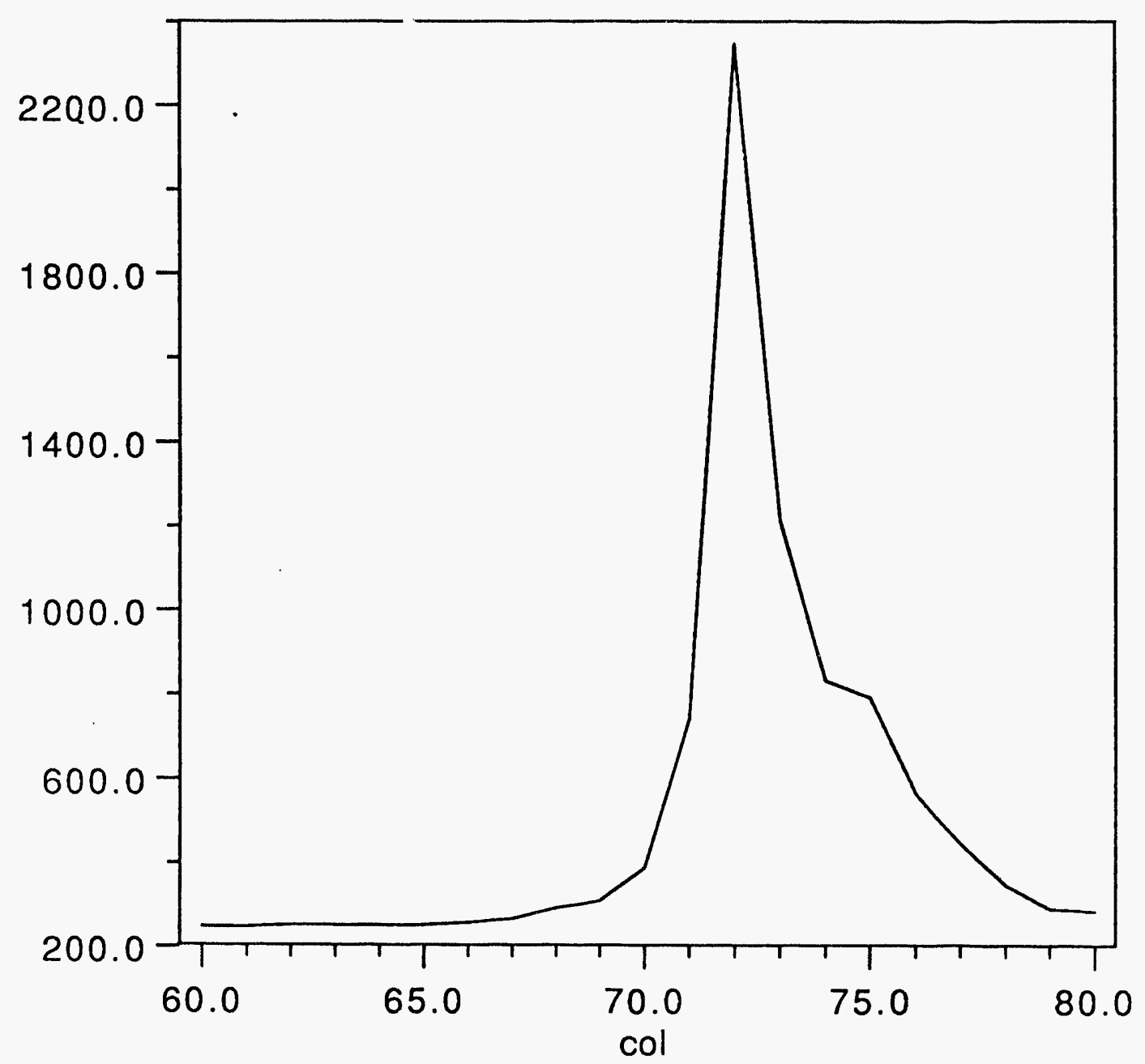

Figure 13 Plot of intensity along row 38 (through peak) for figure 12 data. FWHM of main peak is 2 pixels 

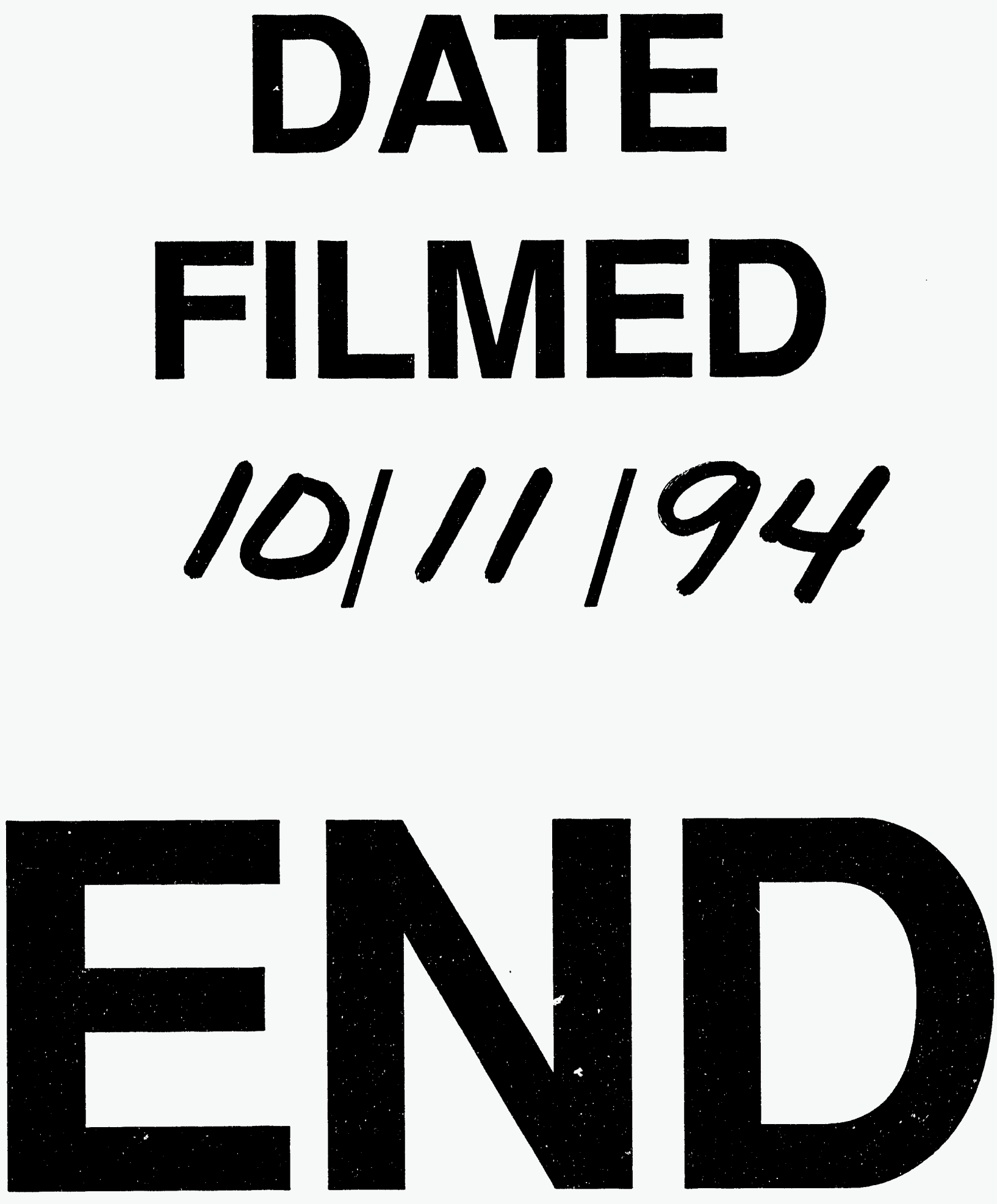
